\title{
Research of Space Network Node Route Planning and Localization in HAPS Space
}

\author{
${ }^{1}$ Hengyang Liu, ${ }^{1,2}$ Xiaoyang Liu, ${ }^{1}$ Chao Liu \\ ${ }^{1}$ School of Computer Science and Engineering, Chongqing University of Technology, Chongqing,400054, \\ China \\ ${ }^{2}$ College of Engineering, The University of Alabama, Tuscaloosa, Alabama,35401, USA \\ 158010342@qq.com; Ixy3103@163.com; xiuwenzheng2000@163.com
}

\begin{abstract}
Compared with the traditional method of detection tracking, space sensor networks for its good character of the traditional tracking methods inadequate. For the localization algorithm and route planning of space sensor network exists a big positioning error in high altitude platform station (HAPS) space communication. Space sensor network communication system is researched. According to the anchor nodes and unknown nodes, location algorithm and node route planning method of HAPS are proposed in this paper. The performance of the node density and location error are simulated and analyzed in HAPS. The simulation results show that the performance of proposed location error is better than the traditional algorithms.
\end{abstract}

Keywords: Network Communication; Location Algorithm; Anchor Node; Mathematical Model

\section{Introduction}

The high altitude platform stations (HAPS) is a new information system that has been developed at an early stage in recent years. Stratospheric HAPS is a key instrumentality for broadband wireless communication, as it could complement and cooperate with satellite and terrestrial communications. So far, in the process of studying and developing of space sensor networks, security has been concentrated less. As a new network, the space sensor network is a multi-discipline, highly intersecting researched hot field, which is of both military and business values. Topology control is one of the most fundamental problems in space sensor networks [1-3], which are the newest technology of information collecting and processing, have a wide range of application including military and business. But the node localization information played a key role in the application of space sensor network. The uplink capacity formula for the HAPS-terrestrial CDMA system is deduced and the capacity of the ring structure and that of the multilayer structure are compared [4-5]. Research of the space sensor network gradually gets the focus from the industrial and academe [6-7]. It has a great application future in the military and civil area. Reducing power consumption to extend network lifetime is one of the most important challenges in designing space sensor networks.

Anchor node positioning usually depends on manual deployment or using GPS. The manual deployment of anchor nodes is not only limited by the network deployment environment. It also severely restricts the 
scalability of networks and applications. With GPS positioning. The cost of anchor nodes will be two orders of magnitude higher than that of ordinary nodes. This means that even if only $10 \%$ of the nodes are anchor nodes, the price of the entire network will increase by 10 times. In addition, the range of positioning accuracy increases with the increase of the density of anchor nodes. When it reaches a certain level, it will not increase again. Therefore, the density of anchor nodes is also one of the important indicators for evaluating the performance of positioning systems and algorithms.

References [8-15] prolonged network lifetime, good scalability and proper load balancing are important requirements for many sensor network applications. Clustering sensor nodes is an effective technique for achieving these goals. Clustering Algorithm based on Node Correlation (CANC) is proposed. CANC utilizes received signal strength, residual energy and connectivity to choose cluster-heads. It takes the node correlation into account to determine cluster members. Analysis and simulation results show preliminarily that, the new CANC algorithm can make cluster-heads well distributed and achieve good performance in terms of system lifetime, scalability and LBF (load balancing factor). Classic clustering algorithms in space sensor networks are studied and find two main reasons causing unnecessary energy consumption, which are fixed operation periods and too much information exchanged in cluster-heads selection. Then an Energy-Efficient Clustering Algorithm (EECA) is proposed, whose kernels are adaptive operation period model and a new cluster-heads selection method [16-18]. Simulation results show that the proposed protocol can adjust operation period adaptively and reduce the information exchanging in choosing cluster-heads, is more energy-efficient and suitable for space sensor network.

A new location algorithm and route planning for use in space sensor network are studied in References [19-20]. References [21-22] presents the analytic and simulation results of the performance of UWB relative location estimation in space sensor networks, and analyze and evaluate the energy consumption models in space sensor networks with probabilistic distance distributions. References [23-24] propose resolving schemes of data collection in space sensor networks of both plane model and linear and nonlinear mathematics model, and proposed a new node route planning method.

The rest of this paper is organized as follows. In Section II, the space sensor network system of HAPS model node route planning is researched. In Section III, the location algorithm and route planning is built up for the space sensor network communication system. In Section IV, simulation results are presented. Finally, conclusions are drawn in Section V.

\section{Node Route Planning}

The space sensor network is drawn in Figure.1. Space sensor network includes a anchor nodes and unknown nodes. It can be implemented with a laser or microwave communication between them. It is a challenging problem about the location algorithm. The traditional location algorithm of SCAN [25] for space sensor network can be seen in Fig.1.

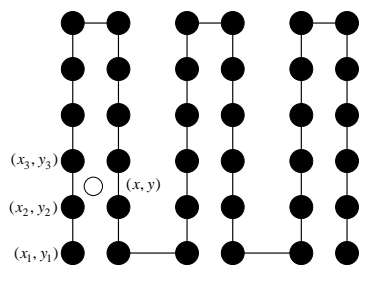

Figure 1. SCAN location algorithm 
Hengyang Liu, Xiaoyang Liu, Chao Liu; Research of Space Network Node Route Planning and Localization in HAPS Space, Transactions on Networks and Communications, Volume 6 No. 3, J une (2018); pp: 10-18

As can be seen from Fig.1. First, in one direction (such as scanning from outside to inside), the sequence of the requested service is sequentially accessed during the scanning. Scan backwards when scanning to the innermost service sequence. Note here that assuming the innermost layer is track 0 . The sequence of the innermost request service is no. 5. After visiting no. 5, it is reversed, and there is no need to sweep back. In the better understanding of the elevator process, when the elevator accesses people, it is clear that the lowest level is no one. It will not go any further. We suppose some node that is the beacon node, the position is $\left(x_{1}, y_{1}\right)$, one node is the unknown node, the position is $(x, y)$, We suppose the distance from the beacon node to unknown node is $d_{1}$, so we can get:

$$
\begin{aligned}
& \left(x_{1}-x\right)^{2}+\left(y_{1}-y\right)^{2}=d_{1}^{2} \\
& \text { s.t. } 0 \leq x \leq l_{1}, 0<y \leq l_{2}
\end{aligned}
$$

The traditional location algorithm of CIRCLES for space sensor network [20-21] is shown in Fig.2.

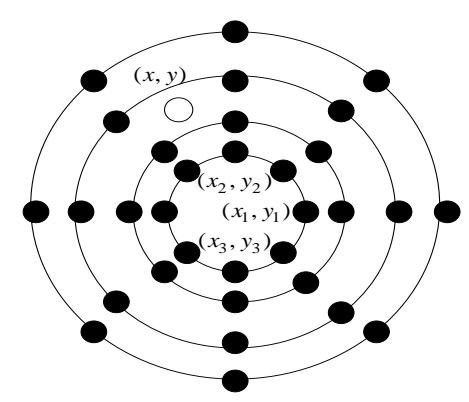

Figure 2. CIRCLES location algorithm

The relationship of multilateral localization of the unknown node is shown in Fig.3.

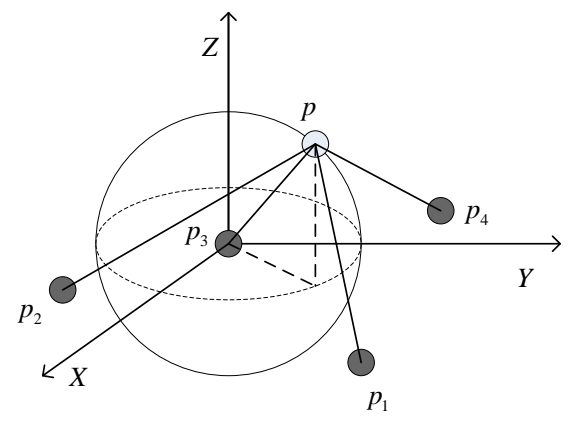

Figure 3. Multilateral localization of the unknown node

There are two beacon nodes in the space sensor network system. The position of beacon node $C\left(x_{2}, y_{2}, z_{2}\right)$ , $A C=d_{2}$, the mathematics model can be denoted as 


$$
\left\{\begin{array}{l}
\left(x_{1}-x\right)^{2}+\left(y_{1}-y\right)^{2}+\left(z_{1}-z\right)^{2}=d_{1}^{2} \\
\left(x_{2}-x\right)^{2}+\left(y_{2}-y\right)^{2}+\left(z_{2}-z\right)^{2}=d_{2}^{2} \\
y=0 \text { or } y=l_{1} \text { or } z=l_{2} \\
\text { s.t. } 0 \leq y \leq l_{1}, 0<z \leq l_{2}
\end{array}\right.
$$

There are three beacon nodes in the space sensor network system, the mathematics model can be expressed as

$$
\left\{\begin{array}{l}
\left(x_{1}-x\right)^{2}+\left(y_{1}-y\right)^{2}+\left(z_{1}-z\right)^{2}=d_{1} \\
\left(x_{2}-x\right)^{2}+\left(y_{2}-y\right)^{2}+\left(z_{2}-z\right)^{2}=d_{2}{ }^{2} \\
\left(x_{3}-x\right)^{2}+\left(y_{3}-y\right)^{2}+\left(z_{3}-z\right)^{2}=d_{3}{ }^{2} \\
y=0 \text { or } y=l_{1} \text { or } z=l_{2} \\
\text { s.t. } 0 \leq y \leq l_{1}, 0<z \leq l_{2}
\end{array}\right.
$$

The distance of anchor nodes can be calculated as follows:

$$
d_{k j}=\left\{\begin{array}{ll}
{\left[\sum_{i=1}^{l_{k}}\left(s_{k i}-x_{j i}\right)^{\alpha}\right]^{1 / \alpha}} & k=1 \\
{\left[\sum_{i=1}^{l_{k}}\left(s_{k i}-x_{j\left(i+l_{k-1}\right)}\right)^{\alpha}\right]^{1 / \alpha}} & k>1
\end{array} \quad k=1,2, \ldots, M ; j=1,2 .\right.
$$

\section{Location and Route Planning Algorithm}

For space sensor network node location, due to the location of the mobile node insert uncertain nodes, so the inserted into the virtual anchor nodes, which helps to limit the location error. The node system model is shown in Fig.4.

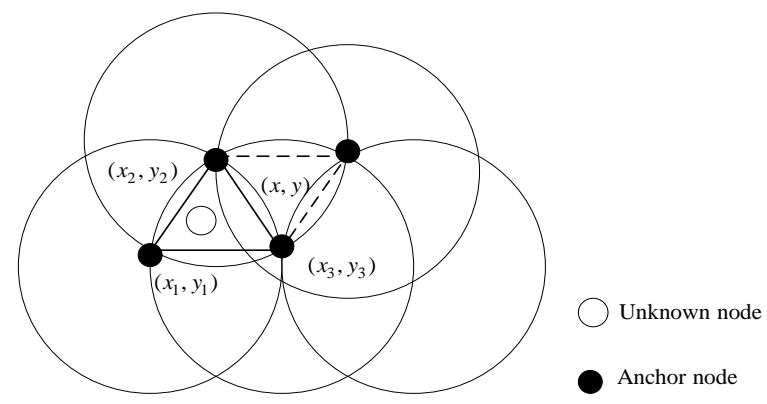

Figure 4. The node system model

The node movement route plan is obtained. So we can get:

$$
\left\{\begin{array}{l}
x=x_{0}+r \times t \times \cos (2 \pi t+\varphi) \\
y=y_{0}+r \times t \times \sin (2 \pi t+\varphi)
\end{array}\right.
$$

Similarly, in the triangle, we can get:

$$
\cos \theta=\frac{\left(d_{2}^{2} / 4\right)+d_{1}^{2}-d_{0}^{2}}{d_{1} d_{2}}
$$

Combined with formula (6) and (7), $d_{0}$ can be expressed as: 


$$
d_{0}=\frac{\sqrt{2 d_{1}^{2}+2 d_{3}^{2}-d_{2}^{2}}}{2}
$$

The total path length can be expressed as

$$
D=\sum_{\mathrm{t}=1}^{20} \sqrt{r^{2}+4 r^{2} \pi^{2} t^{2}+4 r^{2} t \sin (4 \pi t)+4 r^{2} t \cos (4 \pi t)}
$$

The flow chart of space sensor network node location is shown in Fig.5.

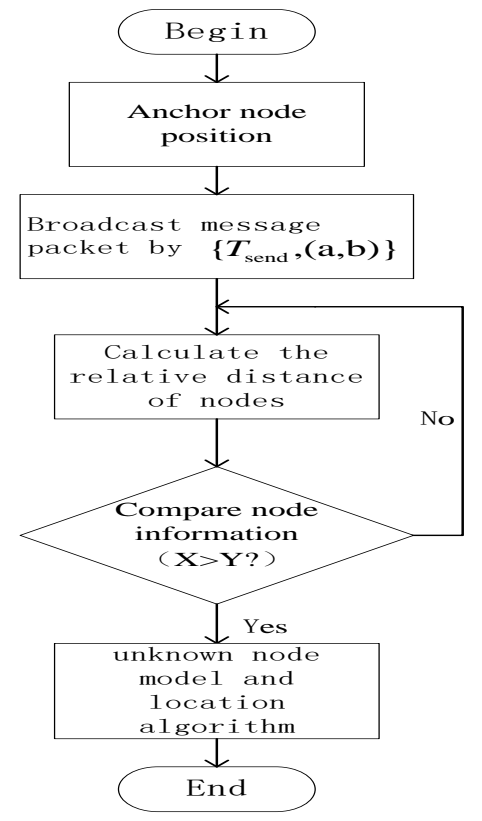

Figure 5. The flow chart of location

Where, the anchor node broadcast message is shown in Fig.6.

We assume that the received packet position is triangle.

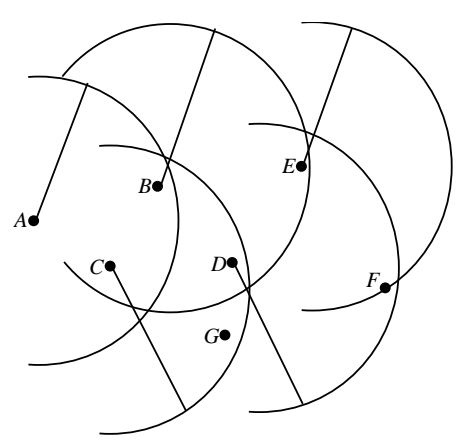

Figure 6. The anchor node of broadcast message

Node relative positioning and the radial error can be expressed respectively:

$$
\text { error }_{\text {avg }}=\frac{\sum_{i=1}^{n} \sqrt{\left(x_{i}-x_{i}^{\prime}\right)^{2}+\left(y_{i}-y_{i}^{\prime}\right)^{2}+\left(z_{i}-z_{i}^{\prime}\right)^{2}}}{n \times R} \times 100 \%
$$




$$
\text { error }_{x}=\frac{\sum_{i=1}^{n} a b s\left(x_{i}-x_{i}^{\prime}\right)}{n \times R} \times 100 \%
$$

\section{Simulation Analysis}

Considering the 150 nodes to be mounted on the random uniform topology. The relation between node radius and location error is shown in Fig.7.

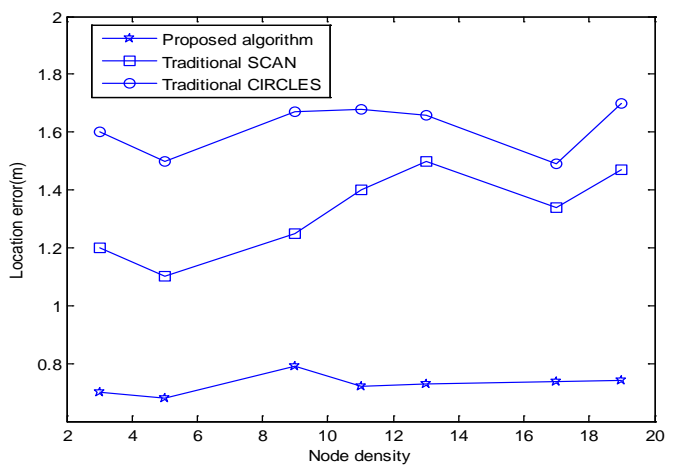

Figure 7. Relation between node density and location error

As can be seen from Fig.7.The location error performance of proposed space sensor network node location algorithm is better than traditional SCAN and CIRCLES. The relation between node density and location proportion is shown in Fig.8.

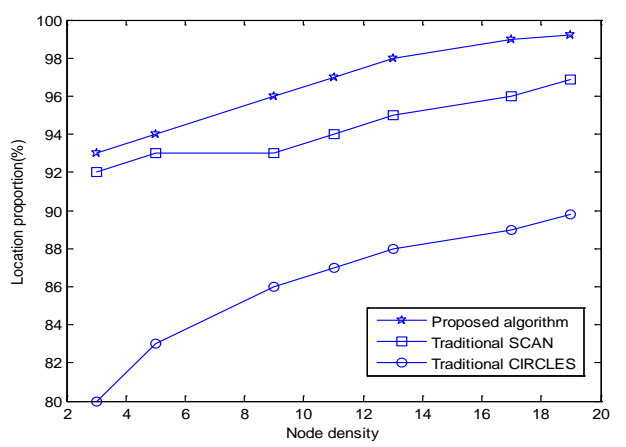

Figure 8. Relation between node density and location proportion

As can be seen from Fig.8. The location proportion performance of proposed space sensor network node location algorithm is better than traditional SCAN and CIRCLES methods. The relation of movement speed of nodes and location error is shown in Fig.9. 


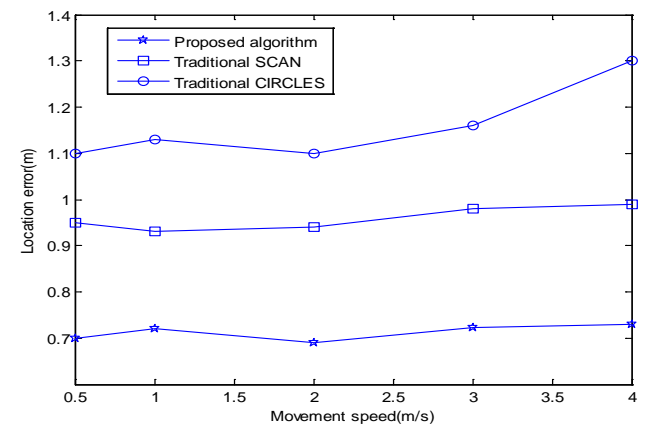

Figure 9. Relation of movement speed and location error

As can be seen from Fig.9. With the increase of movement speed of nodes, the shows different changing tendency along with different location error. In all, the proposed algorithm is better than the traditional SCAN and CIRCLES methods. Location time under different movement speed is shown in Fig.10.

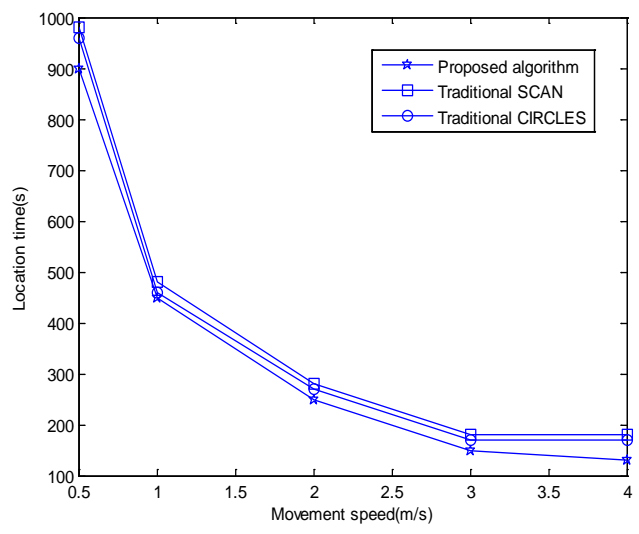

Figure 10. Node density and location error

As can be seen from Fig.10, the location time is different when the movement speed is different. With the increase of movement speed, the location time is becoming less.

\section{Summary}

Based on the analysis of the space sensor network, some conclusions are obtained. First of all, the space sensor network communication system is set up. Then, location algorithm and node route planning of space sensor network are proposed. Some mathematics model is built according to the space sensor network communication system. Lastly, mathematics model and location algorithm and route planning method are simulated. The performance of the proposed location algorithm and route planning method is better than the traditional methods.

\section{ACKNOWLEDGMENT}

The paper was supported by Science and Technology Research Program of Chongqing Municipal Education Commission(KJ1600923,KJ1709206),Young Fund Project of Humanities and Social Sciences Research of Ministry of Education of China (16YJC860010, 15YJC790061,16JDSZ2019),Natural Science Foundation of 
China $(61571069,61501065,61502064,61503052)$,Social Science of Humanity of Chongqing Municipal Education Commission(17SKG144),National Social Science Fund of China West Project(17XXW004).2018 Chongqing Science and Technology Commission Technology Innovation and Application Demonstration (Social Livelihood General) Project(cstc2018jscx-msyb0364).The author Xiaoyang Liu thanks for the financial support from CSC (China Scholarship Council)(201608505142).

\section{REFERENCES}

[1]. Marcus.L.Braga,Alyson.J.Santos, "Anytime route planning with constrained devices,"Computers \& Electrical Engineering, vol.54, pp.53-67,2016.

[2]. Jian An,Ling Qi, Xiaolin Gui,Zhenlong Peng, "Joint design of hierarchical topology control and routing design for heterogeneous wireless sensor networks,"Computer Standards \& Interfaces, vol.51, pp.63-70,2017.

[3]. A.Xenakis,F. Foukalas,G.Stamoulis, "Cross-layer energy-aware topology control through Simulated Annealing for WSNs,"Computers \& Electrical Engineering, vol.56, pp.576-590,2016.

[4]. Rachid Beghdad,Mohamed Abdenour Hocini,Narimane Cherchour, Mourad Chelik, "PEAS with Location Information for coverage in Wireless Sensor Networks,"Journal of Innovation in Digital Ecosystems,vol.3, no.2,pp.163-171,2017.

[5]. Dang Thanh Hai,Le Hoang Son,Trong Le Vinh, "Novel fuzzy clustering scheme for 3D wireless sensor networks,"Applied Soft Computing, vol.54,pp.141-149,2017.

[6]. Amar Kaswan,Kumar Nitesh,Prasanta K. Jana,"Energy efficient path selection for mobile sink and data gathering in wireless sensor networks,"AEU-International Journal of Electronics and Communications,vol.73,pp. 110-118,2017.

[7]. Reem E.Mohemed,Ahmed I. Saleh,Maher Abdelrazzak, Ahmed S. Samra, “Energy-efficient routing protocols for solving energy hole problem in wireless sensor networks,"Computer Networks, vol.114,pp. 51-66,2017.

[8]. Amit Sarkar, T. Senthil Murugan ,"Routing protocols for wireless sensor networks,"Alexandria Engineering Journal,vol.55,no.4, pp.3173-3183, 2016.

[9]. Guangqiang Chen, Bingyan Chen,Pengfei Li, Peng Bai, Chunqun Ji,“Study of aerodynamic configuration design and wind tunnel test for solar powered buoyancy-lifting vehicle in the near-space,"Procedia Engineering,vol.99,pp.67-72,2015.

[10]. Guanhua Wang,Shanfeng Zhang,Kaishun Wu,et al,"TiM: fine-grained rate adaptation in wLANs,"IEEE Transactions on Mobile Computing,vol.15,no.3,pp. 748-761,2015.

[11]. Jie Hu,Lie-Liang Yang,Lajos Hanzo,“Distributed Multistage cooperative-social-multicast-aided content dissemination in random mobile networks,"IEEE Transactions on Vehicular Technology, vol.64, no.7,pp.30753089, 2015.

[12]. Muhammad Aslam, Ehsan Ullah Munir,M. Mustafa Rafique, Xiaopeng Hu, “Adaptive energy-efficient clustering path planning routing protocols for heterogeneous wireless sensor networks,"Sustainable Computing: Informatics and Systems, vol.12, pp.57-71,2016. 
Hengyang Liu, Xiaoyang Liu, Chao Liu; Research of Space Network Node Route Planning and Localization in HAPS Space, Transactions on Networks and Communications, Volume 6 No. 3, June (2018); pp: 10-18

[13]. Yatish K.Joshi, Mohamed Younis, “Restoring connectivity in a resource constrained WSN,"Journal of Network and Computer Applications, vol. 66, pp.151-165,2016.

[14]. Bibin Varghese,Nidhin Easow John, S. Sreelal, Karthika Gopal, “Design and Development of an RF Energy Harvesting Wireless Sensor Node (EH-WSN) for Aerospace Applications,"Procedia Computer Science, vol.93, pp.230-237,2016.

[15]. Nouha Baccour,Anis Koubaa,Habib Youssef,Mario Alves, "Reliable link quality estimation in low-power wireless networks and its impact on tree-routing,"Ad Hoc Networks, vol.27,pp.1-25,2015.

[16]. Juan J. Galvez, Pedro M. Ruiz, “Joint link rate allocation, routing and channel assignment in multi-rate multichannel wireless networks,"Ad Hoc Networks, vol.29,pp.78-98,2015.

[17]. Ajinkya Rajandekar;Biplab Sikdar,"A survey of MAC layer issues and protocols for machine-to-machine communications,"IEEE Internet of Things Journal,vol.2, no.2,pp.175-186, 2015.

[18]. Rodolfo Feick,Mauricio Rodríguez,Luciano Ahumada,et al,“Achievable gains of directional antennas in outdoor-indoor propagation environments," IEEE Transactions on Wireless Communications,vol.14, no.3,pp.1447-1456,2015.

[19]. Prasenjit Chanak,Indrajit Banerjee,R. Simon Sherratt, “Energy-aware distributed routing algorithm to tolerate network failure in wireless sensor networks,"Ad Hoc Networks, vol.56, no.1,pp.158-172,2017.

[20]. Felipe Núñez,Yongqiang Wang,David Grasing, “Pulse-coupled time synchronization for distributed acoustic event detection using wireless sensor networks,"Control Engineering Practice,vol.60, pp.106-117,2017.

[21]. Kejiang Xiao, Rui Wang,Tun Fu, Jian Li, Pengcheng Deng, “Divide-and-conquer architecture based collaborative sensing for target monitoring in wireless sensor networks,"Information Fusion, vol.36, pp.162-171,2017.

[22]. Mohammad Shokouhifar,Ali Jalali, "Optimized sugeno fuzzy clustering algorithm for wireless sensor networks,"Engineering Applications of Artificial Intelligence,vol.60, pp.16-25,2017.

[23]. Zhibo Wang,Honglong Chen,Qing Cao,Hairong Qi,Zhi Wang, Qian Wang.Achieving location error tolerant barrier coverage for wireless sensor networks,"Computer Networks,vol.112,pp.314-328,2017.

[24]. Demin Gao,Haifeng Lin, Xiaofeng Liu, “Routing protocol for k-anycast communication in rechargeable wireless sensor networks,"Computer Standards \& Interfaces, vol.43, pp.12-20, 2016.

[25]. Reem E.Mohemed,Ahmed I.Saleh,Maher Abdelrazzak, Ahmed S.Samra, “Energy-efficient routing protocols for solving energy hole problem in wireless sensor networks,"Computer Networks, vol.114,pp.51-66,2017. 